\title{
Acceso y uso de las TIC en la Universidad Nacional Autónoma de México: el caso de la Biblioteca Central
}

Access and use of th ICT in the Universidad Nacional Autónoma de México: the case of the central library

\author{
Silvia González Marín (1), Marisa González González (2), Omar Hernández Pérez (3), \\ Gloria Adriana Hernández Sánchez (4), Juan Romeo Rojas Rojas (5), Verónica Soria Ramírez (6), \\ Ruth Hanako Takayanagui García (7) y Julio Zetter Leal (8)
}

(1) Dirección General de Bibliotecas, Edificio de Biblioteca Central, Circuito Interior s/n México D. F., Coyoacán C.P.04510 marins@servidor.unam.mx, (2) marisag@dgb.unam.mx,

(3) consulta@dgb.unam.mx, (4) ahdz@dgb.unam.mx, (5) jromeror@dgb.unam.mx,

(6)documentos@dgb.unam.mx, (7) seriunam@dgb.unam.mx, (8) jzetter@dgb.unam.mx

\begin{abstract}
Resumen
Se presenta un diagnóstico del uso de los recursos electrónicos de información del Sistema Bibliotecario de la Universidad Nacional Autónoma de México (UNAM), basado en una encuesta aplicada a estudiantes de licenciatura que acudieron a la sala de consulta de la Biblioteca Central al inicio del ciclo escolar 2006-2007. A partir de los resultados se diseñan propuestas para mejorar el uso y aprovechamiento de esos recursos

Palabras clave: Alfabetización informativa. Biblioteca universitaria. Educación superior. Estudios de usuarios. Internet. Universidad Nacional Autónoma de México. Recuperación de información. TIC. Recursos de información.
\end{abstract}

\section{Introducción}

La Universidad Nacional Autónoma de México (UNAM) fue la primera Universidad del Nuevo Mundo, se fundó en 1551 por decreto real de Carlos V, bajo el nombre de Real Universidad de México, y desde 1929 fue denominada Universidad Nacional Autónoma de México (UNAM, 2001).

La UNAM es hoy en día la institución de educación superior y de investigación más importante del país, ya que contribuye con más del $50 \%$ a la investigación científica nacional y forma importantes cuadros profesionales en prácticamente cualquier área del conocimiento. Su oferta educativa esta conformada por dos modalidades de bachillerato, 81 licenciaturas y 40 programas de posgrado, con una población estudiantil en el ciclo 2005-2006 de 286484 alumnos en todos los niveles (UNAM, 2006).

\begin{abstract}
This paper presents a diagnosis on the use of the electronic information resources provided by the Library System of the National Autonomous University of Mexico (UNAM), based upon a survey applied to UNAM undergraduated students that used the Central Library reference service at the beginning of the 2006-2007academic year. The results will support the design of strategies and initiatives aimed to improve the access and use of electronic information resources.
\end{abstract}

Keywords: Higher education. Information literacy. Information retrieval. Information resources. Information technologies. Internet. National Autonomous University of Mexico. University library. User studies.

Como apoyo a sus tareas sustantivas docencia, investigación y extensión de la cultura- la UNAM ha desarrollo y aplicado las TIC para fortalecerlas, lo cual le ha valido ser una de las universidades más importantes y destacadas de Iberoamérica, de acuerdo a los ranking mundiales. En el año 2006, el suplemento Higher Education del rotativo inglés The Times ubica a la UNAM en el lugar 74 de entre las 200 mejores universidades del mundo; el Centro de Información y Documentación Científica (de España) la ubica en lugar 81 como la de mayor actividad y presencia en Internet; el Institute of Higher Education de la Shanghai Jiao Tong University la coloca en el rango 151 de las 200 mejores universidades del mundo y el SCImago Research Group la ubica en el $4^{\circ}$. lugar en producción científica de los países iberoamericanos (UIAE, 2007). 
Asimismo, la UNAM es la institución educativa pública que más invierte en TIC, tanto en infraestructura como infoestructura. En este último aspecto, no sólo adquiere sino también ha generado varios sistemas de información y bases de datos que son referente obligado para diversos sectores académicos y profesionales del país. Uno de los propósitos fundamentales del Sistema Bibliotecario de la UNAM (SBU), coordinado por la Dirección General de Bibliotecas (DGB), es que esos recursos sean aprovechados óptimamente por la comunidad universitaria.

La necesidad de conocer el grado de optimización de los recursos que invierte la UNAM en las TIC, la ha conducido a crear mecanismos permanentes de evaluación para medir su desempeño. De esta manera, al crearse en 2005 el Programa Transdisciplinario en Investigación y Desarrollo para Facultades y Escuelas (Chavarría, 2005), cuyo objetivo principal es fomentar la creación de proyectos de investigación, fortalecer la actividad académica y lograr la participación y la convergencia de académicos de escuelas y facultades con los de institutos y centros, la DGB -como responsable de la gestión y provisión de productos y servicios de información especializada-, se suma a una de la líneas de investigación que contemplaba ese programa, la del Megaproyecto sobre Tecnologías para la Universidad de la Información y la Computación.

En el aspecto social de este megaproyecto se gesta el estudio de usuarios denominado Acceso y uso de sistemas electrónicos de información de la Biblioteca Central: estudio de caso, cuyo fin es ofrecer un diagnóstico del uso de los recursos electrónicos de información del SBU por parte de los estudiantes de licenciatura que acuden a la sala de consulta de la Biblioteca Central de la Ciudad Universitaria.

\section{Tecnología y nación}

De acuerdo a las cifras oficiales, para el año 2007 México tendría una población aproximada de 105.8 millones de habitantes, de los cuales 52 millones son hombres $(49,2 \%)$ y 53,7 millones son mujeres $(50,8 \%)$ (CONAPO, página Web).

Algunos indicadores muestran que en México las TIC cobran cada día mayor presencia y que la población y las instituciones públicas y privadas están siendo más impactadas en sus actividades y servicios brindados a través de ellas. En estudios recientes se puede observar el creciente número de computadoras: en 2006 había 5.545.667, de las cuales 2.735.143 tenían conexión a Internet; para 2007 la previsión es lle- gar a 14,8 millones de computadoras, con 8,7 millones conectadas a la red. De las computadoras con acceso a Internet el $55 \%$ están en hogares y el $45 \%$ en empresas e instituciones (AMPICl, 2007). Es notable también el avance de la banda ancha, de 2,6 millones de cuentas el año pasado hoy existen 3,9 millones, que representan el $45 \%$ de las computadoras conectadas a Internet.

Se calcula que en México actualmente hay 22.7 millones de internautas, el $92 \%$ de los cuales considera a Internet el medio más indispensable, incluso por arriba del teléfono celular (AM$\mathrm{PICl}, 2007)$.

De acuerdo con datos de 2004, aproximadamente dos de cada tres personas $(63 \%)$ que usan computadora lo hacen con fines de apoyo escolar y una de cada tres (36\%) lo utiliza en sus actividades laborales. La opción de entretenimiento también es utilizada aunque en menor medida, ya que una de cada 6 personas $(16,3 \%)$ señaló utilizar la computadora con fines lúdicos. Aún así, es seguro que en México la penetración de Internet en la población está aún seriamente restringida: sólo uno de cada 10 hogares cuenta con acceso a la red mundial (Inegi, 2005).

Al igual que en otras partes del mundo, en México el impacto de las TIC en el proceso educativo nacional también ha ido adquiriendo una fuerte presencia. Por ejemplo, ya para 2003 dieciséis universidades mexicanas reportaban tener en algunas carreras planes y programas de estudio en la modalidad de educación virtual.

Dentro de este sistema se encuentran integradas las universidades públicas, federales y estatales, entre las cuales ocupa un papel relevante la Universidad Nacional Autónoma de México, que está desarrollando actualmente 47 programas de estudio en línea (ya sea carrera, diplomado, curso de educación continúa, especialidad o posgrado) y varias bibliotecas y colecciones digitales y un vasto número de publicaciones electrónico-digitales, sistemas de información y bases de datos.

\subsection{TIC en la UNAM}

El uso de las TIC en la UNAM se remonta al año 1958, cuando adquirió la primera computadora electrónica digital que se ponía en operación en América Latina, se trataba de una IBM 650, la primera computadora que fabricaba en serie la misma firma (Pinsanty, 2000). El final de los años 60's y el principio de la década de los 70's marcaron para la UNAM la etapa de inicio de las comunicaciones telefónicas y de datos. Es en 
ese periodo cuando se realizan las primeras conexiones de teletipos hacia una computadora central, utilizando líneas telefónicas de cobre, de la recién instalada red telefónica dentro de la institución. En 1987 se establece la primera conexión a la Red Académica de C ó BITNET, mediante enlaces telefónicos, desde la Ciudad Universitaria (CU) hasta el Instituto Tecnológico de Estudios Superiores de Monterrey y de ahí hasta San Antonio, Texas, en los EUA.

En 1990 la UNAM fue la primera institución en América Latina que se incorpora a la red mundial Internet, ante la necesidad de integrar los diferentes servicios y recursos de cómputo como soporte de desarrollo eficiente para la investigación y la docencia. En 1992 surge la Red Integral de Telecomunicaciones de la Universidad Nacional Autónoma de México RedUNAM.

La RedUNAM atiende diariamente a 400 mil usuarios, de los cuales alrededor de 170 mil son parte de la Universidad, y los 230 mil restantes corresponden a conexiones de centros de institutos de investigación, universidades públicas y privadas de todo el país, hospitales y dependencias de gobierno con las que la UNAM colabora. El número de los servicios que la UNAM brinda en promedio mensual asciende a más de 14 millones; en éstos se incluyen los hits al $W e b$, los correos electrónicos enviados y recibidos, FTP y Telnet.

De suma importancia y para potenciar la dinámica académica y de investigación de los universitarios, en 2006 se puso en servicio la Red Inalámbrica Universitaria (RIU) en CU. Dicha Red, cubre los espacios de reunión estudiantil y académica más frecuentes como aulas, bibliotecas, cafeterías y explanadas, operando en más de 55 instalaciones del Campus CU. A partir de marzo de este año se extendió a otras instalaciones de la zona metropolitana de la Ciudad de México -facultades de Estudios Superiores Acatlán, Aragón, Cuautitlán, Iztacala y Zaragoza- (Vela Capdevila, 2007). El acceso a Internet inalámbrico de la RIU es un complemento de la ya existente RedUNAM, que sustituye e integra a las diversas redes inalámbricas locales, que han sido construidas y operadas en los últimos años, aumentando su rendimiento $\mathrm{y}$ seguridad.

Nuestra Universidad es líder en supercómputo y está a la vanguardia en la materia, el 16 de enero del año en curso, el Rector Dr. Juan Ramón de la Fuente puso en marcha el equipo de supercómputo KanBalan. En el ámbito internacional, KanBalam es la número 126 en la clasificación de las 500 supercomputadoras más rápidas en el mundo, la 44 en cuanto a sitios académi- cos y la 28 respecto a las supercomputadoras instaladas en universidades (UNAM, 2007).

En 2006 la UNAM contó con alrededor de 55 mil computadoras personales, de la cuales el $90 \%$ estuvieron conectadas en red. En seis años, se logró casi duplicar la cifra de equipos conectados, al pasar de 25 mil en el año 2000 a 48 mil 500 en 2006. Cerca del $70 \%$ de sus usuarios fueron alumnos, profesores e investigadores. (Vela Capdevila, 2007).

\subsection{Los recursos de información en la UNAM}

Las colecciones documentales de las bibliotecas de la UNAM abarcan desde los incunables hasta los vanguardistas recursos electrónicos de información en todas las disciplinarias del saber humano, dicha riqueza documental se refleja en la prestación de una amplia gama de servicios.

La infraestructura tecnológica desarrollada en la Universidad propició el surgimiento de los recursos de información electrónica para apoyar sus labores sustantivas. En el año 1966 se crea la Dirección General de Bibliotecas (DGB), como órgano coordinador de un sistema bibliotecario, integrado en 2006 por 142 bibliotecas.

La DGB consciente de las necesidades de información de su comunidad, ha desarrollado colecciones, servicios y recursos de información, que reflejan por una parte los acervos institucionales, y por la otra, adquiere producción científica de vanguardia tanto a nivel regional como internacional, lo que ha convertido a sus servicio en referente obligado en su comunidad y en diversos entornos académicos nacionales.

En un primer momento, 1978, la necesidad de contar con un sistema automatizado que apoyara a los procesos técnicos propició el desarrollo de una base de datos, origen del actual catálogo denominado LIBRUNAM, que es el catálogo por antonomasia de la UNAM. Posterior a este esfuerzo, se desarrollaron otras bases de datos como Tesiunam - catálogo de tesis de los egresados de la institución en todos los niveles-y la coordinación de Seriunam -catálogo hemerográfico nacional. Mención especial merecen dos bases de datos especializadas en revistas latinoamericanas, denominadas Clase y Periódica, creadas por el Centro de Información Científica y Humanística $(\mathrm{CICH})$, actualmente fusionado a la DGB. Clase fue creada en 1975 y Periódica en 1978; en sus inicios fueron concebidas como índices bibliográficos de documentos publicados en revistas editadas en la región; se publicaron trimestralmente hasta 1997, cuando se decidió continuarlas solamente como bases de datos en línea, con actualización diaria 
y difusión vía Internet. A partir del año pasado se incluyó el acceso al texto completo de aproximadamente un $10 \%$ de los artículos.

El avance tecnológico que se dio en la década de los años 80 del siglo pasado propició la implementación de nuevos servicios de recuperación de información. Es así como en 1987 se inició el servicio de búsqueda bibliográfica en bancos de datos en disco compacto en el Departamento de Consulta de Biblioteca Central (BC) con una colección de 17 bases. Para el año 1989 se consolidó el servicio, aumentando su colección a 39 títulos. El crecimiento de esta propicia que para 1992 se implemente una Red Novell con 6 estaciones de trabajo. Esta red fue la primera en su tipo en el Sistema Bibliotecario de la UNAM (UNAM, 1996).

En el año 1993 la BC se conecta a RedUNAM a través de fibra óptica, posteriormente, en 1995 se desarrolla la primera página web de la DGB.

El 2 de diciembre de 1996, la UNAM incursiona formalmente (si bien desde 1993 ya hay antecedentes) en el ámbito de la revista electrónica. El Director de Elsevier en América Latina y la UNAM, a través del $\mathrm{CICH}$ firmaron un convenio por tres años (1996-1999) para acceder a la versión electrónica sus publicaciones, al año siguiente la revista electrónica penetra e impacta substancialmente los servicios bibliotecarios a nivel mundial y en ese mismo año es cuando la publicación electrónica accede a la UNAM (Lugo \& Orozco, 2003 p. 140)

A partir de febrero de 2003 , se gestó el proyecto que organiza, integra y entreteje la información electrónica que se adquiere (por compra o acceso gratuito), además de la producida por la Universidad, a través de la denominada Biblioteca Digital <http://bidi.unam.mx>; desde entonces a la fecha, se han ido desarrollando diferentes tareas encaminadas a la conformación de la Biblioteca Digital de la UNAM.

La inversión de recursos económicos, tecnológicos y humanos destinados para proveer uno de los más vanguardistas sistemas de recuperación de información nos obliga a conocer y diagnosticar el grado de aprovechamiento de dichos recursos. Esta motivación es rescatada en el presente trabajo, donde se analiza el conocimiento y uso de 10 recursos que provee la UNAM a su comunidad (1):

1. Librunam, catálogo de libros de las bibliotecas que conforman el sistema bibliotecario; para diciembre de 2006 incluía 948.568 registros bibliográficos. La UNAM tiene más de 5.741. 700 volúmenes de libros en todo su SBU.
2. Tesiunam, catálogo de tesis que presentan los egresados de la UNAM y de las IES incorporadas para obtener un grado académico. De acuerdo a datos del 2006, tiene 358.206 registros.

3. Seriunam, catálogo colectivo de publicaciones seriadas que se encuentran en el SBU y 236 instituciones de educación superior de México. Hasta diciembre de 2006 ofrecía información catalográfica de 52 mil títulos y de 8.421.934 fascículos.

4. Clase, índice de citas en ciencias sociales y humanidades. Ofrece alrededor de 250 mil registros bibliográficos de cerca de 1.500 revistas de América Latina y el Caribe, especializadas en ciencias sociales y humanidades.

5. Periódica, índice de citas en ciencias y tecnología. Ofrece alrededor de 250 mil registros bibliográficos de cerca de 1500 revistas de América Latina y el Caribe, especializadas en ciencia y tecnología.

6. Hela. Contiene información hemerográfica de las revistas que conforman la colección de la Hemeroteca Latinoamericana. Incluye aproximadamente 3.000 títulos de revistas editadas en América Latina y el Caribe, así como de publicaciones de organismos internacionales de alcance panamericano o iberoamericano.

\section{Tesis electrónicas.}

8. Bases de datos (referenciales y texto completo). La UNAM produce 20 bases de datos, además de adquirir 161 bases de datos multidisciplinarias.

9. Libros electrónicos, acceso a 7.945 mediante suscripción.

10. Revistas electrónicas 19.302 títulos de publicaciones periódicas (incluye proveedores y editores).

Los seis primeros son catálogos producidos por DGB con acceso libre a través de Internet, y los tres últimos son recursos adquiridos y/o producidos por la UNAM, accesibles exclusivamente a través de RedUNAM:

Como parte el sistema bibliotecario destaca la Biblioteca Central, la mayor biblioteca multidisciplinaria de la UNAM. Recibe diariamente 11.000 usuarios, en un amplio horario de servicios, de lunes a domingo de 8:30 hrs a 21:30 hrs, interrumpiendo sus servicios exclusivamente en los dos períodos vacacionales de la institución (julio y diciembre). 
La ubicación privilegiada de la Biblioteca Central dentro del Campus le permite que estudiantes de diversas áreas del conocimiento confluyan en sus instalaciones y hagan uso de sus servicios La sala de consulta electrónica se ha convertido en un área muy concurrida, al ofrecer a la comunidad universitaria el acceso a todos los recursos electrónicos que ofrece la UNAM y a la Red de manera general; se cuenta con 50 computadoras conectadas a RedUNAM y la afluencia diaria es de 400 usuarios.

\section{Estudio sobre acceso y uso de las TIC en la UNAM}

\subsection{Diseño de la encuesta}

Debido a que nuestro objetivo es conocer el acceso y uso de los recursos electrónicos se decidió que la población idónea a entrevistar era aquella que al acudir a la sala de consulta electrónica ya tenía cierto bagaje de conocimientos en el uso de la computadora e Internet.

Así mismo, se decidió entrevistar en primera instancia a los estudiantes, por ser ellos quienes hacen mayor uso de la $\mathrm{BC}(2)$.

Con el objeto de obtener información de primera mano, se aplicó una encuesta a 501 estudiantes y pasantes de licenciatura que entre el 28 de agosto y el 6 de septiembre de 2006 acudieron a la sala de consulta de la Biblioteca Central. Una vez en la sala, la elección del entrevistado fue aleatoria y la entrevista se realizó de persona a persona.

El cuestionario "Acceso y uso de Recursos Electrónicos. Perfil de los estudiantes que acuden a la Biblioteca Central" consta de 37 reactivos, divididos en cuatro rubros: Datos generales (sexo, edad, carrera, semestre 0 año, entre otros); Acceso a recursos electrónicos (incluye aspectos como los lugares donde acceden a Internet, dónde o con quién aprendieron a usar la computadora e Internet, formas y medios de acceso, buscadores, navegadores, etc); Uso de los recursos electrónicos (horario, lugares y frecuencia de uso; conocimiento y uso de recursos electrónicos tanto de la UNAM como en general); Datos socioeconómicos (solicitados con la intención de construir estratos socioeconómicos que puedan servirnos para el análisis, con preguntas como escolaridad de los padres, bienes y servicios en el hogar, trabajo del entrevistado, entre otras).

Para este trabajo utilizamos aquellos datos que nos permitieron hacer un diagnóstico del uso que los estudiantes hacen de los recursos electrónicos de información proporcionados por la
UNAM. En una primera parte presentamos algunas características demográficas y escolares de la población entrevistada. Posteriormente describimos ciertas formas de acceder y usar las TIC en particular el Internet y finalmente presentamos un análisis del uso de los recursos electrónicos de información proporcionados por la UNAM.

\subsection{Resultados}

Los estudiantes entrevistados, que en su mayoría son mujeres $(62.5 \%$ y $37.5 \%$ hombres), tienen entre 17 y 54 años, aunque el promedio de edad es de 22 años y la mayoría (56\%) se ubica entre los 20 y 24 años.

Se entrevistó a estudiantes de 44 licenciaturas de las 77 que ofrece la UNAM, las principales fueron Pedagogía (15,6\%), Psicología $(8,2 \%)$, Lengua y literatura hispánica $(7,4 \%)$, Arquitectura $(7,2 \%)$, Historia $(6,8 \%)$ y Derecho $(6 \%)$.

Estas licenciaturas al ser agrupadas por área del conocimiento se distribuyen de la siguiente manera: Área 1 Físico-Matemáticas e Ingenierías, 14\%; Área 2 Biológicas y de la Salud, 12,2\%; Área 3 Sociales, 23,8\%; Área 4 Humanidades y las Artes, $50 \%$.

\subsubsection{Acceso a Internet}

Por acceso a Internet consideramos la posibilidad de consultar información por medio de una computadora conectada a la Red.

Los entrevistados aprendieron a utilizar la computadora principalmente en la escuela $(45 \%)$, aunque un porcentaje importante lo hizo por sí mismo $(27 \%)$ y una quinta parte $(20,4 \%)$ con ayuda de familiares o amigos.

Cuando se les preguntó sobre aprender a navegar en Internet hay un giro interesante, ya que la mitad $(51,3 \%)$ lo hizo por sí mismo; una cuarta parte $(24,4 \%)$ con ayuda de familiares o amigos y sólo un $8,2 \%$ aprendió en la escuela.

Vale la pena subrayar que además de la Biblioteca Central los sitios donde los entrevistados acceden a Internet son los cibercafés $(73 \%)$, su casa $(65 \%)$ y su escuela $(54 \%)$. Sin embargo, cuando se les preguntó cuál es el sitio donde acceden más frecuentemente, la casa se lleva el primer lugar con el $45 \%$, le sigue el cibercafé con $23 \%$ y la B.C con $15 \%$.

\subsubsection{Uso de Internet}

Por uso se entiende la aplicación de habilidades y conocimientos para consultar información cotidianamente en Internet. 
La gran mayoría de los estudiantes $(89 \%)$ utilizan Internet Explorer como navegador principal de Internet y recurren a Google como principal buscador $(75,4 \%)$.

La forma más frecuente de llegar a los sitios de Internet es a través de un buscador $(70,9 \%)$, muy pocos emplean la lista de favoritos (4\%) y sólo el 22,6\% escriben o pegan la dirección de la página.

La información obtenida muestra que la población entrevistada lleva en promedio 5 años con 5 meses utilizando Internet; y el promedio de uso por sesión es de 1 hora con 46 minutos.

Los estudiantes consideran que las principales ventajas de Internet son la cantidad de información y recursos $(48,7 \%)$ y el acceso a información de todo el mundo (33,3\%).

La gran mayoría de los entrevistados ha usado, al menos una vez, los buscadores (99\%) el correo electrónico $(98 \%)$, ha realizado trámites escolares (96\%) y ha navegado en sitios WEB $(86 \%)$; en menor proporción ha consultado bases de datos (59\%), catálogos en línea (54\%), escuchado música (54\%), chateado (50\%) y descargado programas (45\%).

Algunas de esas aplicaciones o acciones además son utilizadas con mucha frecuencia mínimo una vez a la semana-, como es el caso de los buscadores (92\%), el correo electrónico (91\%), la navegación en sitios Web (82\%), oír música $(70 \%)$ y chatear $(60 \%)$.

Como se puede ver, existen actividades dentro de Internet que no tienen una frecuencia constante aunque hayan sido realizadas por prácticamente todos los entrevistados, como es el caso de los trámites escolares, ya que sólo el $14 \%$ señaló efectuarlos una vez a la semana. Otras actividades como oír música y chatear son señaladas sólo por la mitad de la población entrevistada, pero la frecuencia con la que se realizan es alta.

También se les solicitó que autoevaluaran su destreza para realizar determinadas acciones en Internet. En promedio, las actividades mejor calificadas fueron: usar el correo electrónico $(8,8)$, buscar información para trabajos académicos $(8,0)$, buscar información en general $(8,0)$ y realizar trámites escolares $(8,0)$. Las actividades donde se obtuvo menor destreza fueron la participación en grupos de interés $(6,2)$ y la realización de compras o ventas $(6,2)$.

En general encontramos una actitud bastante positiva hacia las nuevas tecnologías, ya que al $59 \%$ no le cuesta trabajo adaptarse a ellas, el
$66 \%$ busca informarse acerca de ellas y el $73 \%$ disfruta utilizándolas.

\subsubsection{Recursos proporcionados por la UNAM}

Sobre los recursos electrónicos de información proporcionados por la UNAM se indagó el conocimiento, el uso, la frecuencia de uso y las razones de no uso, obteniendo como resultado que si bien los libros y revistas electrónicas $(51 \%$ y $50 \%$ ) son los más conocidos (figura 1), el catálogo Librunam se consolida como el más utilizado (figura 2). El 38\% de los entrevistados señaló usarlo y de esos el $62 \%$ manifestó consultarlo mínimo una vez por semana.

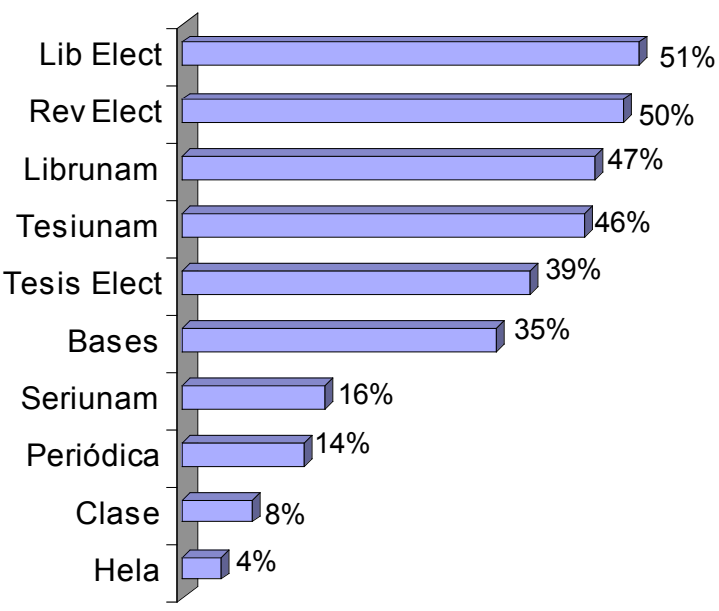

Figura 1. Conocimiento de los recursos electrónicos ofrecidos por la UNAM.

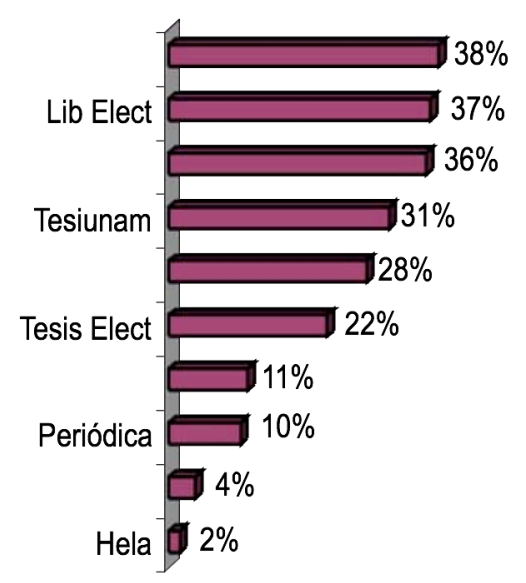

Figura 2. Uso de los recursos electrónicos ofrecidos por la UNAM.

Las bases de datos referenciales especializadas en diversas materias como Periódica, Clase y Hela se ubicaron en los últimos lugares tanto en conocimiento $(14 \%, 8 \%$ y $4 \%$, respectivamente) como en uso ( $10 \%, 4 \%$ y $2 \%$, respectivamente). Lo cual muestra la urgente necesidad de crear 
políticas de promoción, fomento y divulgación de estas bases que son creación de la propia universidad, ya que la información sobre una frecuencia de uso - mínimo una vez a la semana (1VXS) - muestra que entre los usuarios de estas bases la frecuencia constante es alta, incluso mayor que la de otros más conocidos y usados (véase figura 3).

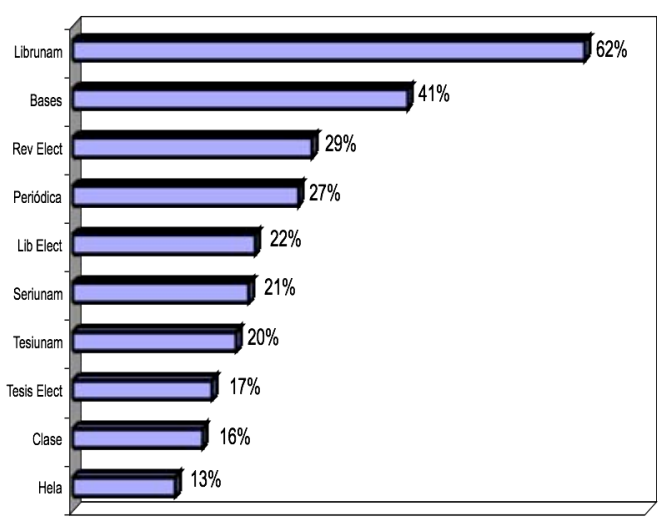

Figura 3. Frecuencia de uso mínimo una vez por semana de los recursos electrónicos ofrecidos por la UNAM.

En los siguientes apartados presentaremos el uso de los recursos analizando algunas características de la población entrevistada; sin embargo, por cuestión de espacio decidimos considerar el conocimiento y uso global, es decir, incluimos en una misma categoría a todos los que conocieran o usaran al menos uno de los diez recursos señalados anteriormente.

Visto de esta manera el conocimiento alcanza al $82 \%$ de los entrevistados y el uso al $70 \%$; de igual forma el $57 \%$ consulta como mínimo una vez por semana al menos uno de los recursos que la UNAM pone a su disposición.

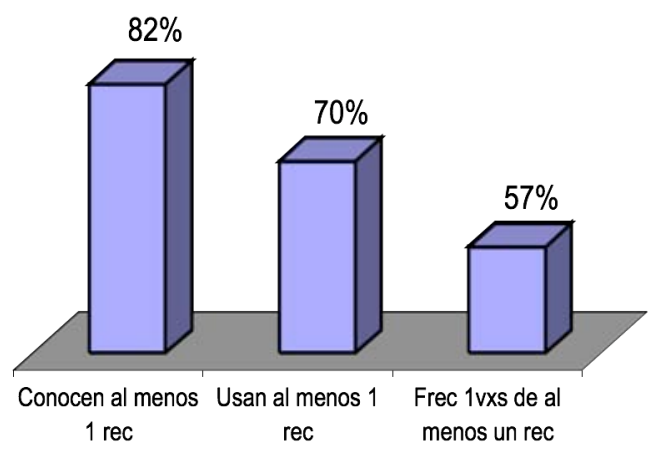

Figura 4. Conocimiento, uso y frecuencia $1 \mathrm{VXS}$ de al menos un recurso ofrecido por la UNAM.

Sexo. En el caso de hombres y mujeres llama la atención que es prácticamente indiferente el sexo del entrevistado, ya que su comportamien- to es casi el mismo. Lo cual indica que una vez en la escuela la brecha entre los géneros disminuye, aunque en determinados aspectos se perfilen ciertas diferencias como una mayor constancia de uso de los recursos en los hombres (véase figura 5 columna frec $1 \mathrm{VXS}$ ).

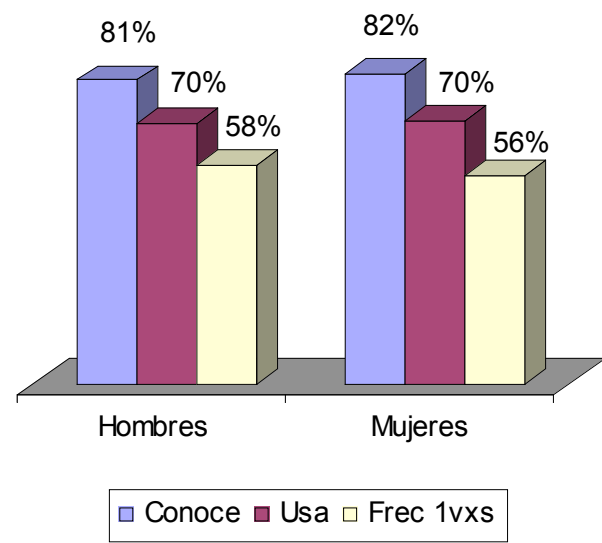

Figura 5. Conocimiento, uso y frecuencia $1 \mathrm{VXS}$ de al menos un recurso ofrecido por la UNAM, según sexo.

Semestre. En el análisis por semestre es claro que conforme van avanzando los estudios crece tanto el conocimiento como el uso, aunque no así la frecuencia. Esta última no sigue una ascendencia como los otros dos aspectos, debido quizá a que la constancia en la búsqueda de información depende de diversos aspectos tales como la materia, el profesor, la disponibilidad de tiempo, etc.

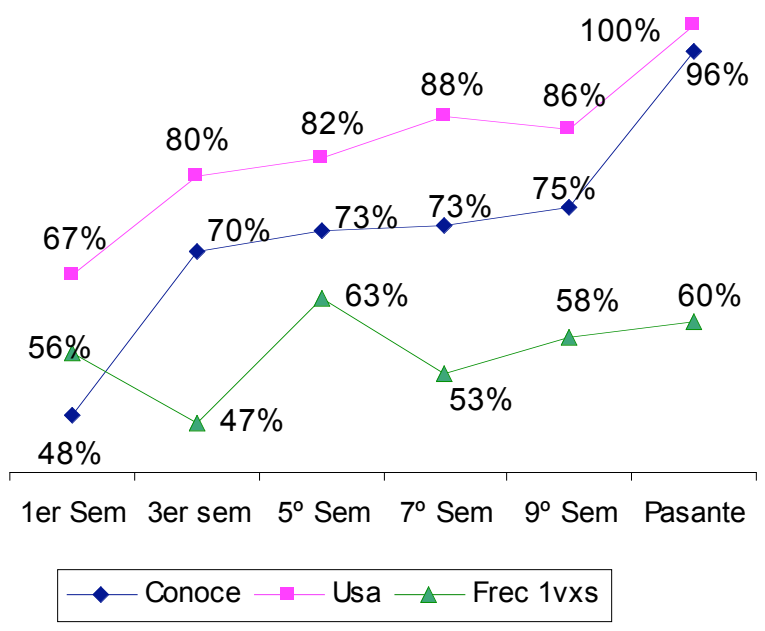

Figura 6. Conocimiento, uso y frecuencia 1VXS de al menos un recurso ofrecido por la UNAM, según semestre. 
Área de conocimiento. Como se ha dicho, la UNAM divide las licenciaturas que imparte en 4 áreas del conocimiento (o del saber). En el área 1 se encuentran las de físico-matemáticas y las ingenierías, en el área 2 las biológicas y de la salud, en el área 3 están las carreras de sociales y en el área 4 las humanidades y las artes.

Sobre esta división temática de las licenciaturas y su utilización de los recursos podemos decir que el mayor conocimiento se encuentra en el área 1 (físico-matemáticas y las ingenierías) y el menor en el área 3 (sociales); el mayor uso está entre los alumnos del área 4 (humanidades y las artes) y el menor nuevamente en el área 3 (sociales). Esta última si bien es la más baja en conocimiento y uso, en frecuencia constante al menos una vez por semana- se ubica en el segundo lugar $(57 \%)$, sólo abajo del área 4 (humanidades y las artes) (61\%).

\section{B) Aspectos tecnológicos y socioeconómicos}

Las características demográficas y escolares analizadas anteriormente nos permiten comprender mejor el conocimiento y uso de los recursos por parte de los estudiantes que acuden a la sala de consulta de la BC. Sin embargo, para completar el panorama, consideramos importante incluir algunas otras que tuvieran relación con el acceso y uso de Internet, como: tiempo que lleva usando Internet y autoevaluación de la destreza; y algunas otras relacionadas con su condición socioeconómica: contar con computadora e Internet en casa, condición de trabajar del entrevistado y escolaridad de los padres.

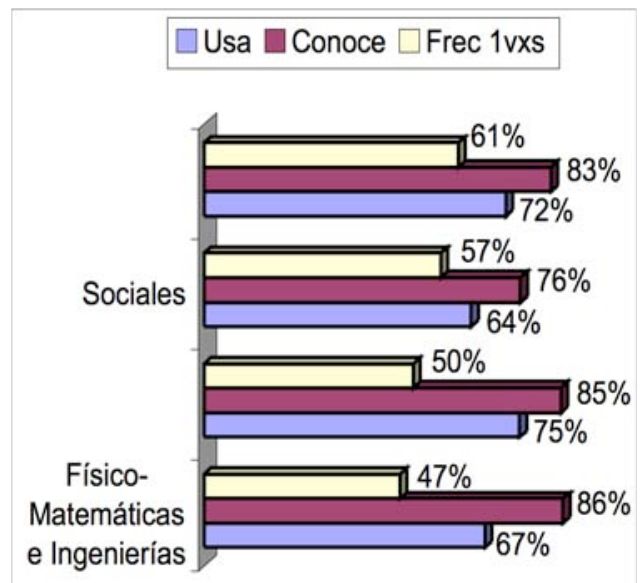

Figura 7. Conocimiento, uso y frecuencia $1 \mathrm{VXS}$ de al menos un recurso según área del saber.

Tiempo de uso y autoevaluación de destreza en Internet. Dividimos el tiempo de uso en 3 cate- gorías: los que llevan menos de 5 años usándolo; los que llevan 5 años y los que llevan más de 5 años. Los años de uso de Internet muestran una tendencia en la cual mientras más tiempo se lleva navegando mayor es el conocimiento y el uso, lo cual implica la existencia de cierta relación y la necesidad de fomentar el acceso. Sin embargo, es importante diferenciar el tipo de sitios a los que se ingresa.

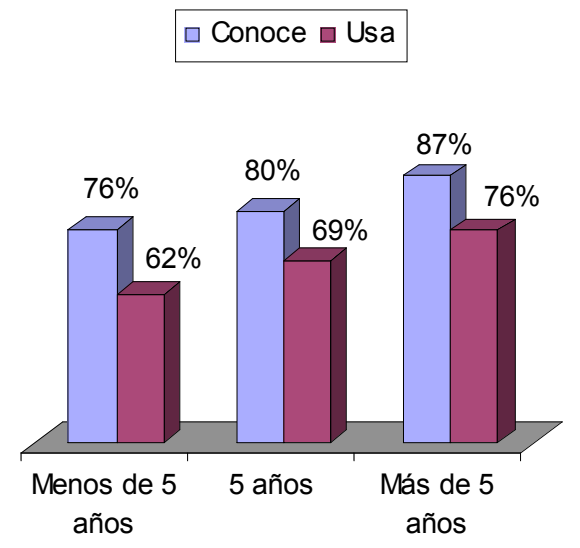

Figura 8. Conocimiento y uso de al menos un recurso ofrecido por la UNAM según tiempo de uso de Internet.

Preguntamos a los alumnos el grado de destreza que consideraban tener en el manejo de determinadas aplicaciones de Internet. Las aplicaciones fueron agrupadas en cuatro categorías:

- entretenimiento (jugar, bajar música, navegar por diversión, etc),

- socialización (chatear, usar el correo electrónico, participar en grupos de interés),

- búsqueda de información (buscar información en general, para realizar trabajos escolares en particular, descargar archivos FTP, descargar programas) y

- trámites (realizar pagos, trámites escolares, vender o comprar).

Para analizar la relación entre los recursos de la UNAM y la autoevaluación de destreza, comparamos la calificación promedio de los que usan los recursos con la calificación de los que no los usan.

En socialización, búsqueda de información y trámites, es mayor la calificación promedio de los que usan los recursos de la UNAM, mientras los que se evaluaron más diestros en entretenimiento son quienes no los usan; es más, ni siquiera los conocen. De lo cual se puede inferir que quienes son asiduos visitantes a sitios de 
entretenimiento pueden alejarse de alternativas, dentro del mismo Internet, que sirven para su desenvolvimiento académico. Situación contraria en otras categorías como socialización, búsqueda de información y realización de trámites.

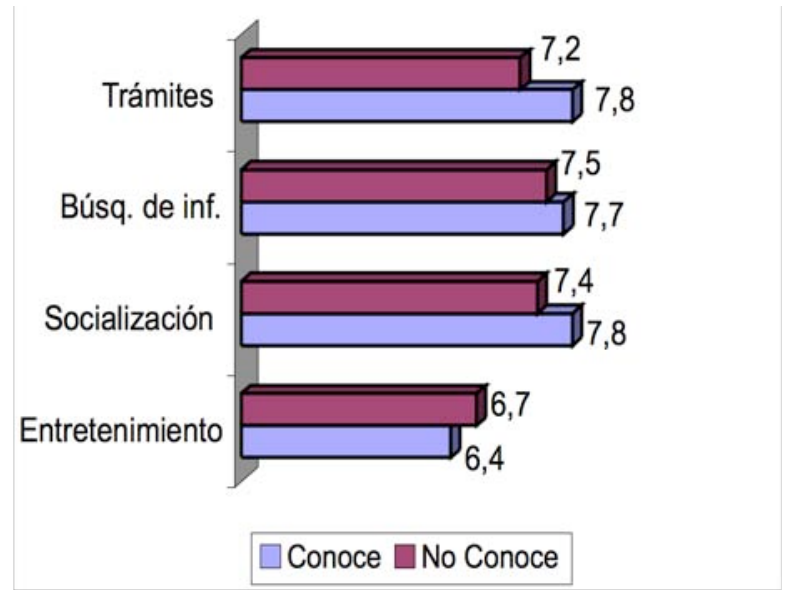

Figura 9. Autoevaluación promedio de destreza en aplicaciones de Internet por condición de conocimiento de algún recurso ofrecido por la UNAM

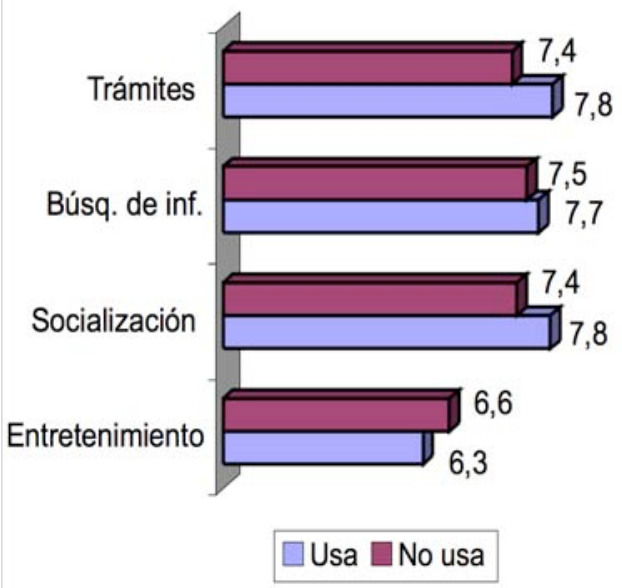

Figura 10. Autoevaluación promedio de destreza en aplicaciones de Internet por condición de uso de algún recurso ofrecido por la UNAM

Computadora conectada a Internet en casa. Partiendo de cierta lógica, consideramos que contar en casa con una computadora conectada a Internet podría estar relacionado con un mayor uso de los recursos electrónicos proporcionados por la UNAM; sin embargo, aunque existe esa tendencia, la diferencia entre los que tienen y no computadora conectada a Internet en casa no es tan grande como podría esperarse, sobre todo en frecuencia de uso constante. Lo cual indica que finalmente ese elemento de brecha digital no es determinante entre estudiantes universitarios, pues éstos cuentan con alternativas de consulta, como las salas de cómputo de sus escuelas y/o facultades, los cibercafés y la Sala de Recursos Electrónicos de la Biblioteca Central, entre otras.

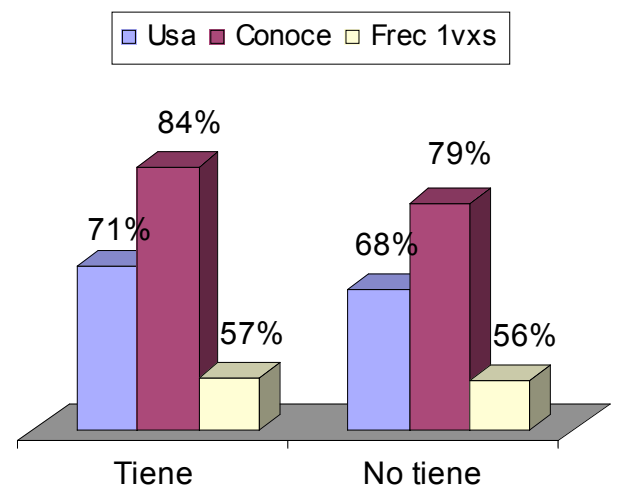

Figura 11. Conocimiento, uso y frecuencia 1VXS de al menos un recurso ofrecido por la UNAM según condición de tener en casa computadora conectada a Internet

Trabajo del entrevistado. El trabajo también podría ser otro elemento que permitiera o apoyara la consulta de los recursos electrónicos; no obstante, aunque sí existe mayor conocimiento, uso y frecuencia entre los que trabajan, la diferencia tampoco es relevante (véase figura 12).

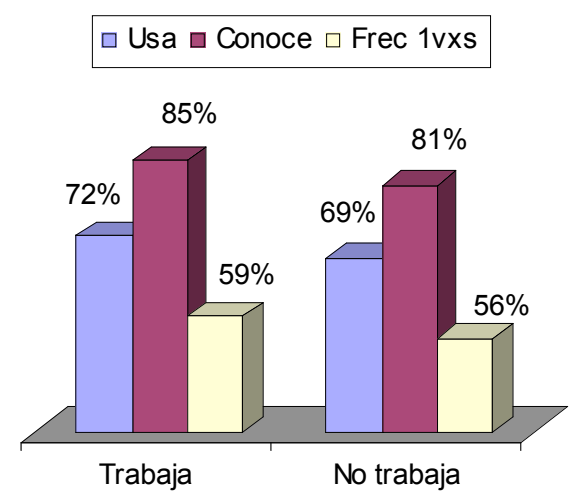

Figura 12. Conocimiento, uso y frecuencia $1 \mathrm{VXS}$ de al menos un recurso ofrecido por la UNAM según condición de trabajo del entrevistado

Escolaridad de los padres. Uno de los aspectos que se considera influyen en el conocimiento y uso de las nuevas tecnologías es la escolaridad de los padres del entrevistado. El sistema educativo mexicano se divide en tres niveles: básico, medio superior y superior. El nivel básico abarca 6 años de primaria y tres años de secundaria; el medio superior tres años de bachi- 
llerato y carreras técnicas, mientras que la educación superior comprende los estudios profesionales y los prosgrados. En este caso tomamos la escolaridad del progenitor con mayor nivel de estudios. Agrupamos los niveles de escolaridad en dos: secundaria o menos y postsecundaria. En el estudio se puede observar que efectivamente hay cierta relación entre escolaridad de los padres y el uso de los recursos ya que los hijos con alguno de sus padres con postsecundaria tienen mayor conocimiento y uso de los recursos proporcionados por la UNAM; sin embargo, la frecuencia de uso es incluso mayor entre los de menor escolaridad. Lo cual quizá muestre que los jóvenes con padres de bajos estudios una vez que hacen uso aprovechan en mayor proporción la oportunidad.

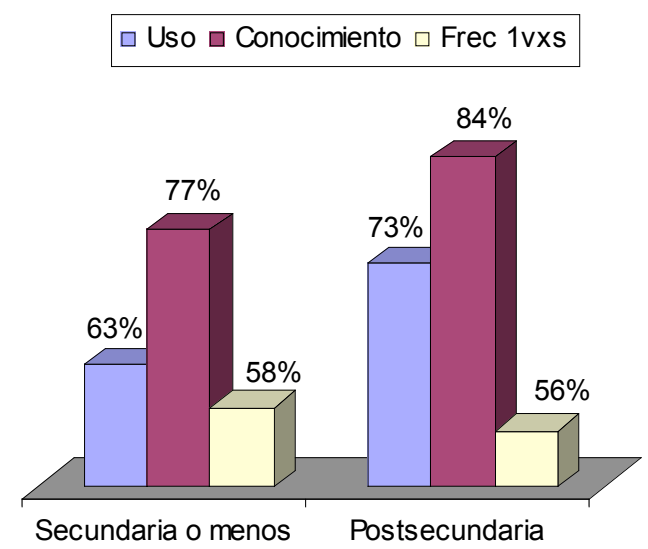

Figura 13. Conocimiento, uso y frecuencia $1 \mathrm{VXS}$ de al menos un recurso según nivel escolar del padre o madre con mayor estudio

\begin{tabular}{|c|c|c|c|c|c|}
\hline \multirow{2}{*}{ Recursos ofrecidos por la UNAM } & \multicolumn{5}{|c|}{ Razones de no uso } \\
\cline { 2 - 6 } & $\begin{array}{c}\text { No me } \\
\text { interesa }\end{array}$ & $\begin{array}{c}\text { No se usarlo/ } \\
\text { uso complicado }\end{array}$ & No me sirve & No lo he necesitado & No tengo acceso \\
\hline Librunam & $23 \%$ & $35 \%$ & $14 \%$ & $7 \%$ & $5 \%$ \\
\hline Tesiunam & $23 \%$ & $31 \%$ & $27 \%$ & $11 \%$ & $1 \%$ \\
\hline Seriunam & $42 \%$ & $42 \%$ & $4 \%$ & $0 \%$ & $4 \%$ \\
\hline Bases & $27 \%$ & $46 \%$ & $11 \%$ & $0 \%$ & $3 \%$ \\
\hline Libros Elect & $26 \%$ & $33 \%$ & $10 \%$ & $4 \%$ & $10 \%$ \\
\hline Revistas Elect & $43 \%$ & $25 \%$ & $12 \%$ & $3 \%$ & $7 \%$ \\
\hline Clase & $19 \%$ & $38 \%$ & $10 \%$ & $5 \%$ & $10 \%$ \\
\hline Periodica & $28 \%$ & $24 \%$ & $11 \%$ & $11 \%$ & $11 \%$ \\
\hline Tesis Elect & $30 \%$ & $20 \%$ & $22 \%$ & $12 \%$ & $4 \%$ \\
\hline Hela & $43 \%$ & $21 \%$ & $14 \%$ & $0 \%$ & $0 \%$ \\
\hline Global & $37 \%$ & $35 \%$ & $21 \%$ & $11 \%$ & $6 \%$ \\
\hline
\end{tabular}

Tabla I. Razones de no uso de los recursos electrónicos ofrecidos por la UNAM.

Razones de no uso. Un elemento indispensable para la definición de estrategias es saber las razones que los entrevistados que conocen los recursos de la UNAM dan sobre el no uso de los mismos. Si bien existen algunas diferencias en las razones dadas en cada recurso (véase tabla I), los tres principales argumentos son: no me interesa (37\%), no se usarlo/uso complicado $(35 \%)$ y no me sirve (21\%). Lo cual significa que es necesario tomar medidas no sólo para dar a conocer los recursos que la UNAM proporciona, sino también explicar lo que ofrece y hacerlos "amigables" para la consulta.

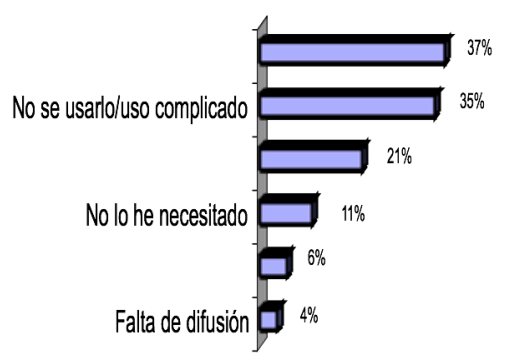

Figura 14. Razones de no uso de los recursos electrónicos ofrecidos por la UNAM. 


\section{Consideraciones finales}

Las TIC irrumpieron en la vida moderna afectando a todas las facetas y ámbitos de la sociedad, las universidades no podían quedar ajenas a esta realidad. Poco a poco fueron introduciéndose en las áreas de gestión de las mismas, para posteriormente incursionar en la docencia e investigación. Si bien, en un primer momento la incorporación de las TIC fue lenta -y azarosa- en la actualidad se ha insertado como un elemento esencial de la vida universitaria.

Desde mediados de la década de los noventa la palabra Internet forma parte del vocabulario cotidiano y los estudiantes mexicanos no escapan de tal realidad mundial, ya que conocen, utilizan, se informan y se actualizan de las novedades, bondades y servicios que la Red les ofrece; en otras palabras, disfrutan de navegar, buscar y socializar en este medio.

Sí bien las actividades de entretenimiento ocupan un lugar preponderante en los usos de los encuestados, un alto porcentaje utiliza Internet como complemento de sus actividades académicas.

Los datos obtenidos sobre el conocimiento y uso de los recursos proporcionados por la UNAM nos indican que los estudiantes entrevistados recurren a los medios electrónicos e impresos de forma indiscriminada; mientras que en conocimiento predominan los libros y revistas electrónicas, en el uso Librunam es la principal base de datos seguida de libros electrónicos.

Por tipo de documento, el libro, ya sea en soporte impreso o electrónico, continúa siendo el principal referente de apoyo a la formación académica. Las tesis, sobre todo al final de la licenciatura, se convierten en fuente indispensable de consulta. Las revistas, a pesar de ser un soporte documental que registra los avances científicos de vanguardia, no son lo suficientemente explotadas durante la formación profesional escolarizada.

De hecho, encontramos que en general los recursos ofrecidos por la UNAM no se aprovechan de forma óptima, pues los alumnos entrevistados mostraron bajo conocimiento y uso de ellos. Una posible explicación en el caso de las bases de datos producidas por la UNAM es que no identifican la denominación precisa del recurso aunque hagan uso de él. El manejo iconográfico de la página Web de la DGB permite ingresar a estas bases de datos sin tener que saber exactamente el nombre del recurso. Para las bases de datos suscritas una limitante es el desconocimiento de las mismas, el idioma de origen y su especialización temática.
Por otro lado, al relacionar el conocimiento y uso de los recursos ofrecidos por la UNAM con determinadas características demográficas, escolares, tecnológicas y socioeconómicas de los entrevistados, identificamos la existencia de cuatro niveles de correspondencia. En un primer lugar, factores como ubicarse en los últimos grados de estudio, llevar más tiempo navegando en la Red, considerarse más diestros en actividades internáuticas de socialización, trámites escolares y búsqueda de información y tener algún padre con un nivel escolar superior a la secundaria, parecen ser elementos de peso para un mayor conocimiento y uso de los recursos electrónicos ofrecidos por la UNAM.

En un segundo nivel encontramos factores como el hecho de que el entrevistado trabaje o tenga computadora conectada a Internet en casa. Sin embargo, si bien se alcanzan porcentajes superiores entre los que conocen y usan que entre los que no conocen y usan, las diferencias son poco marcadas, por lo que difícilmente podríamos identificar una incidencia.

En otro nivel se encuentran el sexo del entrevistado y el área de saber al que pertenecen, factores que no muestran tener influencia para alcanzar mayor conocimiento y uso de los recursos electrónicos. Quizá en esta descripción podríamos dejar fuera al área de sociales, ya que al compararla con las otras tres presenta niveles de conocimiento y uso más bajos.

En un último nivel tenemos el hecho de que autoevaluarse más diestros en actividades de entretenimiento parece influir negativamente, ya que la calificación promedio fue mayor entre los que no conocen y usan los recursos electrónicos proporcionados por la UNAM que entre los que señalaron hacer uso de ellos.

Finalmente, cabe destacar las principales razones que dan los estudiantes que conocen pero no usan los recursos electrónicos para explicar su falta de consulta: en primer lugar, señalan que no les interesa; en segundo, que no saben usarlo o que su uso es complicado; y, en tercer lugar, que no les sirven. Lo anterior muestra la necesidad de contar con acciones y programas institucionales sobre alfabetización informativa, que en la UNAM se encuentran en fase inicial. Adicionalmente, se detecta la necesidad de incrementar los esfuerzos de promoción y difusión de estos recursos, que aunque si se realizan, deben ser más agresivos.

\section{Algunas propuestas}

Los entrevistados tienen como promedio 5 años de uso de Internet, lo que significa que poseen 
cierta experiencia, pero ésta no se refleja en los datos arrojados por la encuesta respecto al uso de los recursos de la DGB. Este tiempo de experiencia pudiera ser aprovechado para alfabetizarlos en el complejo y dinámico mundo de la información. Por lo señalado anteriormente, la DGB ha decidido poner especial énfasis en potenciar y desarrollar las habilidades y capacidades informativas de los estudiantes. Algunas de las estrategias:

1. Efectuar Sesiones de Bienvenida en Biblioteca Central, cuyo objetivo es presentar a los alumnos de licenciatura de primer ingreso los servicios y recursos que la BC ofrece. Se imparten al inicio del ciclo escolar, y son un requisito básico para obtener la credencial de préstamo domiciliario. Ahora estas sesiones se realizarán permanentemente y no solo para alumnos de primer ingreso.

2. Desarrollar el Programa de Habilidades para el Uso de Recursos de Información, conformado por tres módulos: 1) catálogos generales, 2) bases de datos adquiridas por la UNAM y 3) a la carta. Al inicio del ciclo escolar se establece un calendario de sesiones fijas. Ahora se pretende que las sesiones se realicen con más flexibilidad de acuerdo a las posibilidades de horario de los alumnos, y diseñar una estrategia de difusión con mayor alcance.

3. Realizar periódicamente campañas de difusión de los recursos por área temática, que incluyan sesiones informativas y talleres.

4. Crear carteles, trípticos, folletos, entre otros, que de manera permanente estén informando de las novedades en productos y servicios de información.

5. Realizar sistemáticamente diversos estudios que permitan establecer programas y acciones ad-hoc para optimizar el acceso y uso de las TIC en el sistema Bibliotecario de la UNAM.

6. Crear un cuerpo colegiado multidisciplinario, integrado por especialistas en la materia, que establezca los lineamientos y acciones para optimizar el uso de los recursos que ofrece la UNAM.

\section{Notas}

(1) La información sobre el número de registros de los catálogos no está todavía publicada, se obtuvo del Departamento de Procesos Técnicos, del Departamento de Tesis y de la Subdirección de Servicios de Información, de la DGB.
(2) Para complementar el diagnóstico, actualmente se lleva acabo el diseño de una encuesta a profesores de la Institución.

\section{Referencias}

Asociación Mexicana de Internet (AMPICI) (2007). Estudio 2007, usuarios de Internet en México y uso de nuevas tecnologías. http://www.amipci.org.mx/en_los_medios. php? mcmvme=44 (2007-04-16)

Consejo Nacional de Población (CONAPO). Proyecciones de la Población de México 2005-2050.:http://www.cona po.gob.mx/00cifras/5.htm (2007-05-29)

Chavarría, Rosa María (2005). Programa de Investigación para Escuelas y Facultades. // Gaceta UNAM. 3:803 (abril, 2005) p.8.

Instituto Nacional de Estadística, Geografía e Informática (INEGI) [2005]. Estadísticas sobre Disponibilidad y uso de Tecnología de Información y Comunicación en los Hogares, 2005. [en línea] [México] INEGI, 28 p. $<$ http://www.inegi.gob.mx/prod.serv/contenidos/espanol/ catalogo/>

Lugo Hubp, Margarita y Orozco Aguirre Aurelia (2003). La revista electrónica en la UNAM: retos presentes y futuros. // Biblioteca Universitaria. 62 (2003) 138-151

Pinsanty, Alejandro (2000). Contribución de la UNAM al desarrollo de las Tecnologías de Información en México. http://www.tidap.gob.mx/Presentaciones/Paneles\%20y\% 20Conferencias/pisanty.pdf

UIAE (2007) La UNAM en el ranking mundial. // El Financiero (4 de junio 2007). http://www.elfinanciero.com.mx/ ElFinanciero/Portal/cfpages/contentmgr.cfm?docld=61335 \&docTipo $=1$ \&orderby $=$ docid\&sortby $=$ ASC

Universidad Nacional Autónoma de México (UNAM) (2001). Suplemento de los 450 años de la Universidad de México. // Gaceta UNAM. 3481 (septiembre 2001).

Universidad Nacional Autónoma de México (UNAM) (2006). Agenda Estadística $2006 \mathrm{http}: / / \mathrm{www}$. planeacion.unam. mx/agenda/2006/.

Universidad Nacional Autónoma de México (UNAM) (2007). Estrena UNAM la supercomputadora más potente de América Latina. // Banco de Boletines 031. DGCSUNAM (2007). http://www.dgcs.unam.mx/boletin/ bdboletin/2007_031.html (22 de mayo de 2007).

Universidad Nacional Autónoma de México (UNAM) (1996). El Sistema Bibliotecario. México: UNAM, 1996. ISBN 968-36-55679-X $60 \mathrm{p}$.

Vela Capdevila, José Antonio (2007). Se inaugura la III Feria de cómputo. // Gaceta UNAM (7 de mayo de 2007). 